\title{
Distinguishing the contributions of implicit and explicit processes to performance of the weather prediction task
}

\author{
Amanda L. Price \\ Elizabethtown College, Elizabethtown, Pennsylvania
}

\begin{abstract}
Examinations of the cognitive neuroscience of category learning frequently rely on probabilistic classificationlearning tasks - namely, the weather prediction task (WPT) - to study the neural mechanisms of implicit learning. Accumulating evidence suggests that the task also depends on explicit-learning processes. The present investigation manipulated the WPT to assess the specific contributions of implicit- and explicit-learning processes to performance, with a particular focus on how the contributions of these processes change as the task progresses. In Experiment 1, a manipulation designed to disrupt implicit-learning processes had no effect on classification accuracy or the distribution of individual response strategies. In Experiment 2, by contrast, a manipulation designed to disrupt explicit-learning processes substantially reduced classification accuracy and reduced the number of participants who relied on a correct response strategy. The present findings suggest that WPT learning is not an effective tool for investigating nondeclarative learning processes.
\end{abstract}

Responding to environmental events often demands rapid categorization of both familiar and novel stimuli. The learning required for such categorization readily occurs under a variety of circumstances, and it is generally believed that such learning is supported by multiple memory systems (Ashby, Alfonso-Reese, Turken, \& Waldron, 1998; Ashby \& Valentin, 2005; Maddox \& Ashby, 2004; Poldrack et al., 2001; Poldrack \& Packard, 2003; Smith, Patalano, \& Jonides, 1998; but see Lagnado, Newell, Kahan, \& Shanks, 2006, and Nosofsky \& Johansen, 2000, for discussions of a unitary system of categorization). At least one of these systems supports declarative, explicit category learning, ${ }_{1}^{1}$ which requires active memorization and/or hypothesis testing, whereas at least one other system supports nondeclarative, implicit category learning, which develops in a gradual and nonconscious manner.

A number of cognitive, neuropsychological, and neuroimaging studies have supported the existence of multiple category-learning systems (for relevant reviews, see Ashby \& Valentin, 2005; Shohamy, Myers, Kalanithi, $\&$ Gluck, 2008). In these investigations, one of the most commonly used paradigms is the weather prediction task (WPT), a probabilistic classification task designed to assess gradual, incremental learning over many trials (Knowlton, Squire, \& Gluck, 1994). On this task, participants must categorize a set of visually presented cues that are probabilistically related to one of two outcomes and then receive feedback on the accuracy of their response. The probabilistic cue-outcome relationships are thought to disrupt declarative-learning processes (Knowlton, Mangels, \& Squire, 1996; Knowlton et al., 1994; Pol- drack et al., 2001). For this reason, the WPT has been used extensively to examine the neural mechanisms that support nondeclarative learning (Aron, Gluck, \& Poldrack, 2005; Foerde, Knowlton, \& Poldrack, 2006; Poldrack et al., 2001; Poldrack, Prabakharan, Seger, \& Gabrieli, 1999; Shohamy et al., 2004) and to assess the integrity of nondeclarative learning in various neuropsychological populations (Beninger et al., 2003; Eldridge, Masterman, \& Knowlton, 2002; Foerde et al., 2008; Hopkins, Myers, Shohamy, Grossman, \& Gluck, 2004; Kéri, Szlobodnyik, Benedek, Janka, \& Gádoros, 2002; Knowlton et al., 1996; Knowlton et al., 1994).

Although the WPT is commonly considered a measure of nondeclarative learning, neuropsychological evidence suggests that the task may rely on declarative-learning processes. Patients with focal damage of the medial temporal lobe (MTL), who experience a selective deficit of declarative memory, exhibit minimal learning even with considerable practice (Hopkins et al., 2004; Meeter, Myers, Shohamy, Hopkins, \& Gluck, 2006). Furthermore, neuropsychological research with healthy older adults and patients with Parkinson's disease indicates that WPT learning is greater among those with higher levels of executive function (Knowlton et al., 1996; Price, 2005). Executive function is positively associated with explicit category learning but has a negative or null relationship with implicit category learning (DeCaro, Thomas, \& Beilock, 2008; Maddox \& Filoteo, 2001), suggesting that WPT performance depends on explicit processes. Behavioral evidence provides further support for this possibility. Concurrent performance of an attention-demanding 
secondary task, which has previously been shown to disrupt explicit, but not implicit, category learning (Jiménez \& Vázquez, 2005; Waldron \& Ashby, 2001; Zeithamova \& Maddox, 2007), impairs performance on the WPT (Foerde et al., 2006; Foerde, Poldrack, \& Knowlton, 2007; Newell, Lagnado, \& Shanks, 2007). Furthermore, WPT performance is positively associated with participants' awareness of the underlying structure (Foerde et al., 2007; Gluck, Shohamy, \& Myers, 2002; Knowlton et al., 1994; Lagnado et al., 2006; Newell et al., 2007; Price, 2005; Reber, Knowlton, \& Squire, 1996).

In addition, analyses of participants' response patterns have indicated that participants often attain relatively high levels of accuracy through consistent reliance on a simple, verbalizable strategy (Gluck et al., 2002; Meeter et al., 2006; Newell et al., 2007). Specifically, Gluck et al. demonstrated that participants tended to initially rely on a singleton strategy, responding optimally on each of the single-cue patterns but randomly on the other patterns. As the task progressed, participants increasingly adopted a onecue strategy, responding solely on the basis of the presence or absence of a particular cue. After considerable training, some participants began to use a multicue strategy, basing their responses on an integration of the optimal outcome for all four cues. Because the singleton and one-cue strategies are more easily verbalized than the multicue strategy, they may reflect the operation of explicit processes, whereas the multicue strategy reflects the operation of implicit processes (Shohamy et al., 2004). If this is correct, it would suggest that explicit processes predominate during early task performance but that learning increasingly depends on implicit processes as the task progresses.

Consistent with this, neuroimaging evidence has indicated that WPT performance was associated with an initial (e.g., first 50 trials; Poldrack et al., 2001) increase in MTL activation, suggestive of declarative learning. As the task progressed, MTL activity decreased and activation increased in striatal regions, suggesting the emergence of nondeclarative processes (Cohen \& Eichenbaum, 2001; Squire, 1992). This pattern of neural activity led to the conclusion that declarative processes mediated by the MTL (e.g., exemplar memorization) support initial learning on the WPT but that nondeclarative processes mediated by the striatum (e.g., habit learning) are largely responsible for performance improvement as the task progresses (Poldrack et al., 2001; Shohamy et al., 2008).

In the present investigation, this possibility was examined by manipulating the WPT so as to assess the specific contributions of implicit- and explicit-learning processes to performance, with a particular focus on how the contributions of these processes changed as the task progressed. More specifically, Experiment 1 manipulated the delay between categorization response and feedback delivery, a modification previously shown to selectively impact implicit category-learning processes (Maddox, Ashby, \& Bohil, 2003; Maddox \& Ing, 2005). Neurobiological models of category learning (Ashby et al., 1998; Ashby \& Valentin, 2005; Shohamy et al., 2008) argued that nondeclarative learning depends on the immediate delivery of feedback to strengthen cue-outcome associations. The delivery of feedback triggers dopamine signals that alter synaptic connections associated with the immediately preceding stimulus and associated response (Arbuthnott, Ingham, \& Wickens, 2000; Hollerman \& Schultz, 1997; Kerr \& Wickens, 2001; Schultz, 1992). If feedback is delayed, the feedback-mediated dopamine release will modify inappropriate synapses, thereby disrupting implicit learning. Explicit category learning is unaffected by a delay in feedback, presumably because the relevant rule can be verbalized and maintained in working memory until feedback occurs (Maddox et al., 2003; Maddox \& Ing, 2005).

To the extent that WPT learning relies upon implicit processes, a delay in feedback should hamper performance. If implicit learning develops only after participants have completed sufficient training (e.g., 50 training trials; Poldrack et al., 2001), feedback delay should selectively impair later task performance. Alternatively, if the WPT primarily relies on explicit processes, feedback delay should have little impact on performance. In addition, if reliance on a multicue strategy reflects the development of implicit knowledge, delaying feedback should reduce the frequency of such strategy use. Alternatively, if explicit knowledge is sufficient to support the use of a multicue strategy, feedback delay should not affect the distribution of individual response strategies.

Whereas Experiment 1 manipulated the operation of implicit processes in WPT learning, Experiment 2 manipulated the operation of explicit processes by disrupting participants' ability to process feedback. According to neurobiological models of category learning, rule-based explicit learning necessitates attention as participants perform hypothesis-testing processes (Ashby et al., 1998; Maddox $\&$ Ashby, 2004). These processes require time and attention. If the time available to process feedback is limited, one is unable to properly execute the hypothesis-testing process, thereby disrupting explicit category learning (Maddox, Ashby, Ing, \& Pickering, 2004). Implicit learning is unaffected, however, because the reward-mediated synaptic changes presumed to support implicit learning occur in a rapid and relatively automatic fashion. Consistent with these arguments, limiting the time available for feedback processing has been shown to disrupt explicit, but not implicit, category learning (Maddox et al., 2004).

To the extent that WPT learning relies on explicit processes, a disruption to feedback processing should impair classification performance. If explicit processes are necessary only during initial task performance, disrupting feedback should impair initial performance only. In addition, if the singleton and one-cue response strategies reflect explicit knowledge, disrupting explicit processes should reduce participants' tendency to rely on these strategies but should have little impact on participants' use of the multicue response strategy. Alternatively, if implicit knowledge is sufficient to support both simple and multicue strategies, feedback delay should have little impact on strategy use. Finally, if WPT performance primarily reflects implicit processes, participants' awareness of the underlying structure in either experiment should be unrelated to classification accuracy or the particular response strategy in use. 


\section{EXPERIMENT 1}

\section{Method}

\section{Participants}

Thirty-eight undergraduate students participated for partial fulfillment of a class requirement. The participants were randomly assigned to either the immediate-feedback $(n=18)$ or the delayedfeedback $(n=20)$ condition.

\section{Materials}

Stimuli presentation and data collection were completed with E-Prime software (Psychology Software Tools, Pittsburgh) on IBMcompatible computers, equipped with 15 -in. Super VGA monitors. Following the general method of Knowlton et al. (1994), the classification task required that the participants learn which of two possible outcomes (rain or sunshine) would occur on each trial on the basis of a set of visual cues. Each of the four cues was a card, 200 pixels in height and 150 pixels in width, bearing a geometric shape (circles, diamonds, squares, or triangles). The cards were situated horizontally along the center of the screen. One, two, or three cards were displayed on each trial, with 14 possible cue combinations. The sequence of cue combinations that appeared on each trial was randomized for each participant, with the constraints that the same cue combination did not appear twice in succession and that neither outcome occurred more than five times in a row. The two outcomes occurred equally often.

Each cue was associated with an outcome according to a fixed probability. Table 1 lists each cue combination, the frequency with which it appeared in the task, and the probability with which it predicted rain (probabilities taken from Knowlton et al., 1994). Across all 14 cue combinations, ${ }^{2}$ Cue 1 was associated $75 \%$ of the time with rain and $25 \%$ of the time with sun; Cue 2 was associated $56 \%$ of the time with rain and $44 \%$ of the time with sun; Cue 3 was associated $44 \%$ of the time with rain and $56 \%$ of the time with sun; and Cue 4 was associated $25 \%$ of the time with rain and $75 \%$ of the time with sun.

\section{Procedure}

Classification learning. The participants were told that they were being placed in the position of weather forecaster and that their job was to decide whether a given set of cues predicted rain or sunshine. They were told that the relationship between cues and outcomes was complex and that, at first, they would be guessing but would gradually become better at deciding which cues predicted rain or sun. The participants completed 160 classification-learning trials with either immediate or delayed feedback.

The procedure for a typical trial in each condition followed Maddox and Ing (2005): immediate feedback condition, classification stimuli appeared/response/500-msec mask/750-msec corrective feedback/5-sec blank screen intertrial interval; delayed feedback condition, classification stimuli appeared/response/5-sec mask/750-msec corrective feedback/500-msec blank screen intertrial interval. The mask consisted of four rectangles, situated in the positions of the four cue cards, each filled with randomly positioned dots.

Assessment of structure awareness. Upon completion of the WPT, the participants were shown each of the four cards and were asked to indicate whether that card was more strongly associated with rain, with sun, or equally with both. The participants were then informed that each of the four cue cards was related to a given outcome according to a specific probability. The participants were then shown each card by itself and were asked to estimate what percentage of the time rain occurred when that card appeared, regardless of whether it appeared alone or in combination with other cards.

\section{Results}

\section{Classification Accuracy}

A participant was considered to have made an optimal response if he/she selected the outcome that was more strongly associated with the cue combination presented,
Table 1

Probability Structure of the Weather Prediction Task

\begin{tabular}{|c|c|c|c|c|c|c|}
\hline \multirow{2}{*}{$\begin{array}{c}\text { Cue } \\
\text { Pattern }\end{array}$} & \multicolumn{4}{|c|}{ Cue } & \multirow[b]{2}{*}{$P($ appearance $)$} & \multirow[b]{2}{*}{$P($ rain $)$} \\
\hline & 1 & 2 & 3 & 4 & & \\
\hline A & 0 & 0 & 0 & 1 & .133 & .150 \\
\hline B & 0 & 0 & 1 & 0 & .087 & .385 \\
\hline C & 0 & 0 & 1 & 1 & .080 & .083 \\
\hline D & 0 & 1 & 0 & 0 & .087 & .615 \\
\hline E & 0 & 1 & 0 & 1 & .067 & .200 \\
\hline F & 0 & 1 & 1 & 0 & .040 & .500 \\
\hline G & 0 & 1 & 1 & 1 & .047 & .143 \\
\hline $\mathrm{H}$ & 1 & 0 & 0 & 0 & .133 & .850 \\
\hline I & 1 & 0 & 0 & 1 & .067 & .500 \\
\hline $\mathrm{J}$ & 1 & 0 & 1 & 0 & .067 & .800 \\
\hline K & 1 & 0 & 1 & 1 & .033 & .400 \\
\hline $\mathrm{L}$ & 1 & 1 & 0 & 0 & .080 & .917 \\
\hline M & 1 & 1 & 0 & 1 & .033 & .600 \\
\hline $\mathrm{N}$ & 1 & 1 & 1 & 0 & .047 & .857 \\
\hline
\end{tabular}

regardless of the probabilistically determined feedback provided on that particular trial. Cue combinations associated equally with both outcomes were excluded from analysis. Figure 1 illustrates the proportion of optimal responses for each condition as a function of block. An examination of individual participants' data indicated that the majority of the participants in each condition exceeded chance levels of performance (50\% accuracy). A chi-square analysis indicated that the proportion of participants failing to exceed chance in the immediate feedback $(10 \%)$ and the delayed feedback (11\%) conditions did not differ $(p>.8)$.

Each participant's mean accuracy, determined as the proportion of optimal responses, was computed for each block of 40 trials. To examine the development of learning in each condition, a mixed ANOVA was conducted, with block (1-4) as the within-subjects factor and learning condition (immediate vs. delayed feedback) as the betweensubjects factor. Overall, accuracy did not differ between

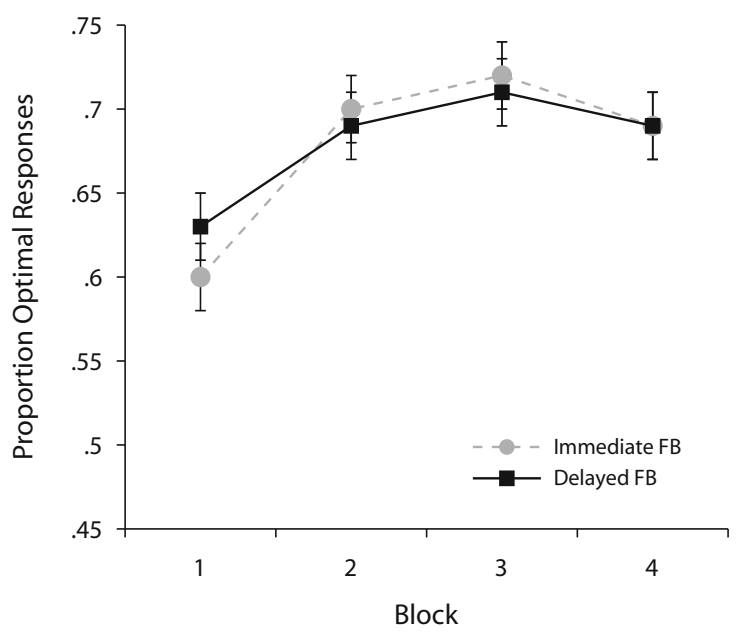

Figure 1. Proportion of optimal responses for each condition in Experiment 1 as a function of block. Error bars represent standard errors of the means. FB, feedback. 
the immediate feedback $(M=.68, S E=.02)$ and the delayed feedback $(M=.68, S E=.02)$ conditions $(p>.9)$. Classification accuracy improved over the course of the task, as indicated by a main effect of block $[F(3,108)=$ $9.00, p<.001]$. The effect of block did not vary by condition $(p>.7)$. These results indicated that learning-related changes in classification accuracy were unaffected by the timing of feedback at any point in the task.

\section{Strategy Analysis}

Gluck et al. (2002; see also Meeter et al., 2006) identified several classes of strategies that participants may use to successfully perform the WPT.

Singleton strategies. The participants relying on this strategy responded with the optimal response for those patterns that presented a single card (Patterns A, B, D, and $\mathrm{H}$ ) and guessed on the remainder of the patterns. The participants were also classified as relying on a singleton strategy if they gave the optimal response only for the two strongly weighted cards (Patterns A and $\mathrm{H}$ ).

One-cue strategies. The participants relying on the one-cue strategy responded solely on the basis of the presence or absence of a single cue, regardless of the other cues presented.

Integrative strategies. ${ }^{3}$ The participants relying on an integrative strategy appeared to learn the optimal outcome for each of the four cues and based their responses on multiple cues. Integrative strategies included (1) singleton plus strategy, in which the participants responded with the optimal outcome on the singleton patterns and patterns presenting either two sun cards or two rain cards (Patterns $\mathrm{C}$ and $\mathrm{L}$ ); (2) the 2 versus 1 strategy, in which the participants responded with the optimal response on each of the singleton and three-card patterns (e.g., two rain cards and one sun card; Patterns G, K, M, and N); (3) the all but $2 v 1$, in which the participants responded with the optimal outcome on all the patterns, except for the three-card patterns; and (4) the multicue strategy, in which the participants responded with the optimal outcome on all patterns.

In addition to these successful strategies, it is possible that the participants may have responded according to strategies that did not support successful performance. Therefore, two inappropriate strategy types were also considered.

Random strategy. The random strategy best fit those participants who were equally likely to have responded sun or rain, regardless of the presented pattern. This "strategy" reflected guessing, rapid switching between strategies, or use of a strategy that more closely approximated random behavior than did any of the other strategies considered.

Wrong strategies. The participants relying on a wrong strategy responded in a consistent but incorrect manner. Several possible incorrect strategies were considered: (1) incorrect singleton strategies, in which the participants consistently responded incorrectly on the single-card patterns and responded randomly on the other patterns; and (2) incorrect one-cue strategies, in which the participants based their responses on the presence or absence of one particular card, but in an incorrect fashion (e.g., respond sun whenever the strong rain card is present; respond rain otherwise).

Following the method outlined by Gluck et al. (2002), ideal data were constructed for each of these strategies and then compared with each participant's response pattern for each block. A least means squares (LMS) procedure was used to calculate the degree to which a participant's data fit with the ideal data for each of the possible strategies. For each block of trials, a participant was classified as following the strategy that produced the greatest fit (lowest LMS) with his/her particular response pattern. Figure 2 illustrates the distribution of response strategies for each condition as a function of block

Frequency of inappropriate strategies. To determine whether the tendency to rely on inappropriate strategies varied across the two conditions, all correct strategies (one cue, singleton, or integrative) were pooled together so that the distribution of random, incorrect, and correct strategies could be compared across conditions. A series of chi-square analyses revealed that the timing of feedback (immediate vs. delayed) did not impact the distribution of correct, incorrect, and random strategies. No differences emerged across conditions for Block $1\left(\chi^{2}=5.12, p>\right.$ $.07)$, Block $2\left(\chi^{2}=0.01, p>.9\right)$, Block 3 (the participants used only correct strategies $)$, or Block $4\left(\chi^{2}=0.92\right.$,
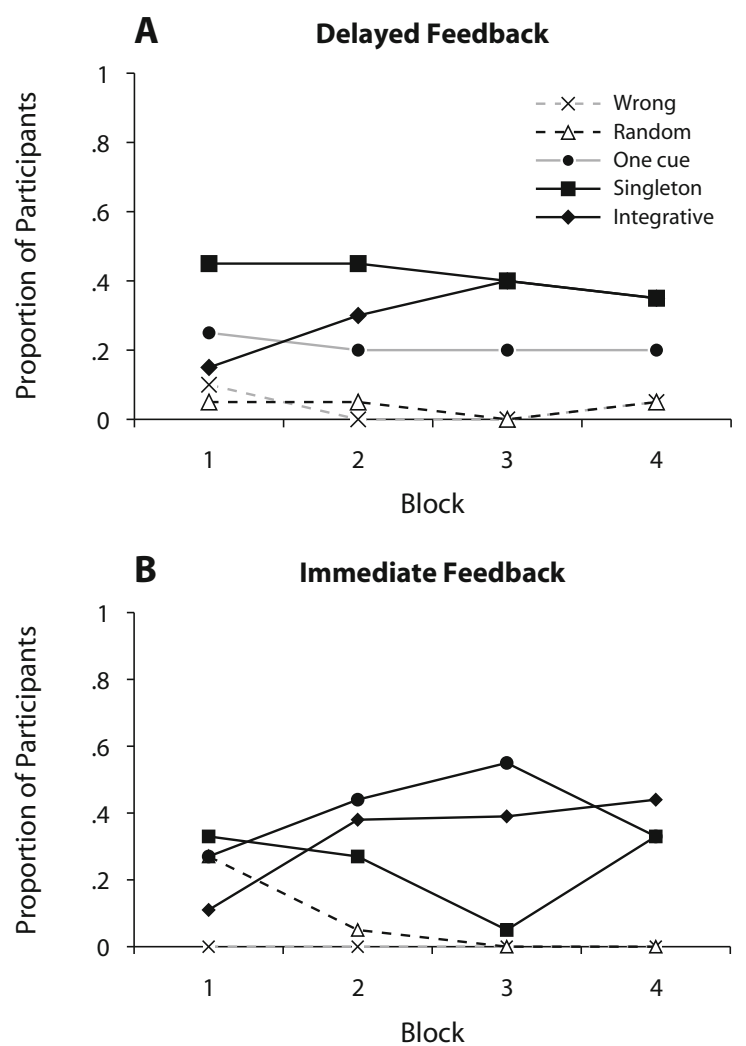

Figure 2. Strategy distribution for the participants in Experiment 1 as a function of block under conditions of delayed feedback (A) and immediate feedback (B). 
$p>$.6). As is shown in Figure 2, the participants in both conditions relied overwhelmingly on correct strategies.

Distribution of correct response strategies. To examine whether the timing of feedback delivery (immediate vs. delayed) had an impact on the distribution of correct response strategies (singleton, one cue, or integrative), a series of chi-square analyses were conducted within each block. ${ }^{4}$ These analyses indicated that the distribution of correct strategies was similar across the two conditions for Block $1\left[\chi^{2}(2, N=30)=0.27, p>.8\right]$, Block $2\left[\chi^{2}(2, N=\right.$ $36)=2.77, p>.2]$, and Block $4\left[\chi^{2}(2, N=35)=2.04\right.$, $p>$.3]. A difference in strategy distribution emerged for Block $3\left[\chi^{2}(2, N=38)=8.00, p<.05\right]$, reflecting the fact that the participants in the immediate-feedback condition were more likely to rely on a one-cue strategy, whereas those in the delay condition were more likely to rely on a singleton strategy; the proportion of participants relying on an integrative strategy did not differ across conditions.

To examine whether the distribution of correct response strategies varied over the course of the task, strategies were reclassified as either simple (one-cue and singleton strategies) or integrative. A series of pairwise block comparisons, using Wilcoxon signed ranks tests, indicated an increased reliance on integrative strategies in Block $3(z=-2.11, p<.05)$ and Block $4(z=-2.0$, $p<.05$ ), relative to Block 1; no other block differences emerged in strategy use $(p>.1)$ for each block comparison. These analyses were then repeated for the distribution of singleton versus one-cue strategies but did not reveal any evidence of a change in simple strategy distribution over the course of the task $(p>.1$ on all block comparisons). Taken together, these data indicated that regardless of the timing of feedback delivery, the participants relied primarily on simple strategies in the early part of the task and increasingly on integrative strategies as the task progressed.

\section{Variations in Accuracy by Strategy Type}

Although the timing of feedback delivery had little impact on the types of strategies that the participants tended to use, it may have affected the way in which they made use of a given strategy. Furthermore, it is likely that WPT performance varied depending on the response strategy in use. To examine these possibilities, mixed ANOVAs were conducted within each block to assess the impact of condition (immediate vs. delayed feedback) and correct strategy type (one cue, singleton, and integrative) on accuracy. A significant effect of strategy type emerged on each of the blocks [Block $1, F(2,24)=4.14, p<.05$; Block 2, $F(2,30)=18.33, p<.001$; Block $3, F(2,32)=$ 3.34, $p<.05$; and Block 4, $F(2,29)=9.21, p<.001]$. Post hoc Games-Howell tests $(\alpha=.05)$ indicated that for Blocks1, 2, and 4, participants who used an integrative strategy performed better than those using a one-cue or singleton strategy. On Block 3, the participants using an integrative strategy outperformed only those using a singleton strategy. Performance did not vary between participants relying on one-cue and singleton response strategies on any of the four blocks. The effect of strategy type on performance did not vary by condition $(p>.2)$, and condition did not impact performance $(p>4)$. To summarize, WPT performance varied considerably with the type of strategy in use, but this effect did not vary as a function of the timing of feedback delivery.

\section{Structure Awareness}

Two measures of structure awareness were created for each participant on the basis of his/her responses during the posttask assessment. The first measure reflected the number of cue-outcome relationships that a participant correctly identified. The second measure reflected the accuracy of a participant's estimates of the individual cue weights and was derived in the following manner. For each of the four cues, the absolute difference was taken between the actual and the estimated probability that rain would follow that cue. The absolute differences for each cue were averaged to create a measure of cue weight awareness; on this measure, lower deviations indicated greater awareness. In Tables 2 and 3, the participants' awareness of the cue-outcome relationships and underlying cue weights, respectively, are presented for each individual cue as a function of feedback condition.

Table 2

Awareness of Cue-Outcome Relationships

\begin{tabular}{llllc}
\hline & \multicolumn{4}{c}{ Cue Card } \\
\cline { 2 - 5 } \multicolumn{1}{c}{ Group } & $\begin{array}{c}\text { Strong } \\
\text { Sun }\end{array}$ & $\begin{array}{c}\text { Weak } \\
\text { Sun }\end{array}$ & $\begin{array}{c}\text { Weak } \\
\text { Rain }\end{array}$ & $\begin{array}{c}\text { Strong } \\
\text { Rain }\end{array}$ \\
\hline Immediate feedback & .80 & .65 & .45 & .80 \\
Delayed feedback & .89 & .39 & .56 & .72 \\
& Experiment 2 & & \\
Short feedback & .69 & .25 & .19 & .38 \\
Long feedback & .56 & .50 & .88 & .56 \\
\hline
\end{tabular}

Note-Each value is the proportion of participants in each group who identified the optimal outcome for that particular cue card.

Table 3 Awareness of Individual Cue Weights

\begin{tabular}{|c|c|c|c|c|}
\hline \multirow[b]{2}{*}{ Group } & \multicolumn{4}{|c|}{ Cue Card } \\
\hline & $\begin{array}{c}\text { Strong } \\
\text { Sun } \\
(.75)\end{array}$ & $\begin{array}{l}\text { Weak } \\
\text { Sun } \\
(.56)\end{array}$ & $\begin{array}{l}\text { Weak } \\
\text { Rain } \\
(.44)\end{array}$ & $\begin{array}{c}\text { Strong } \\
\text { Rain } \\
(.25)\end{array}$ \\
\hline \multicolumn{5}{|c|}{ Experiment 1} \\
\hline \multicolumn{5}{|c|}{ Immediate feedback } \\
\hline$M$ & .72 & .52 & .42 & .21 \\
\hline$S E$ & .06 & .06 & .06 & .04 \\
\hline \multicolumn{5}{|c|}{ Delayed feedback } \\
\hline$M$ & .69 & .49 & .41 & .33 \\
\hline$S E$ & .04 & .06 & .07 & .06 \\
\hline \multicolumn{5}{|c|}{ Experiment 2} \\
\hline \multicolumn{5}{|l|}{ Short feedback } \\
\hline$M$ & .58 & .45 & .58 & .56 \\
\hline$S E$ & .04 & .06 & .06 & .05 \\
\hline \multicolumn{5}{|l|}{ Long feedback } \\
\hline$M$ & .61 & .58 & .32 & .41 \\
\hline$S E$ & .05 & .05 & .04 & .07 \\
\hline
\end{tabular}

Note-The values in parentheses are the actual cue-outcome probabilities. Other values are the means and standard errors for the participants' estimates of the frequency with which each cue card would be followed by rain. 
To determine whether the timing of feedback delivery affected the participants' level of structure awareness, independent samples $t$ tests were conducted on each awareness measure. No difference emerged in cue-outcome awareness for the participants in the no-delay $(M=2.6$, $S E=0.24)$ and delay $(M=2.7, S E=0.25)$ conditions $(p>.8)$. Similarly, the participants in the immediatefeedback $(M=0.2, S E=0.02)$ and delayed-feedback $(M=0.22 ; S E=0.02)$ conditions exhibited comparable levels of cue weight awareness $(p>.7)$. The participants were relatively accurate in their cue weight estimates; a series of one-sample $t$ tests comparing the mean cue weight estimate provided by the participants with the actual cue weight revealed no significant differences for Cue 1 [strong sun; $t(37)=-1.27, p>.2$ ], Cue 2 [weak sun; $t(37)=-1.55, p>.1]$, Cue 3 [weak rain; $t(37)=-0.3$, $p>.7$ ], or Cue 4 [strong rain; $t(37)=0.69, p>.5$ ]. These data indicated that the participants developed a substantial degree of declarative knowledge of the underlying classification structure, regardless of the timing of feedback delivery.

To better understand how structure awareness mapped onto the reliance of particular response strategies, it is worthwhile to determine whether structure awareness differed depending on the strategy in use on the final block of trials. Figure 3 illustrates the two awareness measures as a function of response strategy. Separate ANOVAs were conducted on each measure, with condition (immediate vs. delayed feedback) and response strategy (singleton, one cue, and integrative) as factors. These analyses indicated that cue weight awareness did not differ across conditions $(p>.3)$ or response strategies $(p>.7)$; the interaction also was not significant $(p>4)$. Cueoutcome awareness, by contrast, varied with the type of
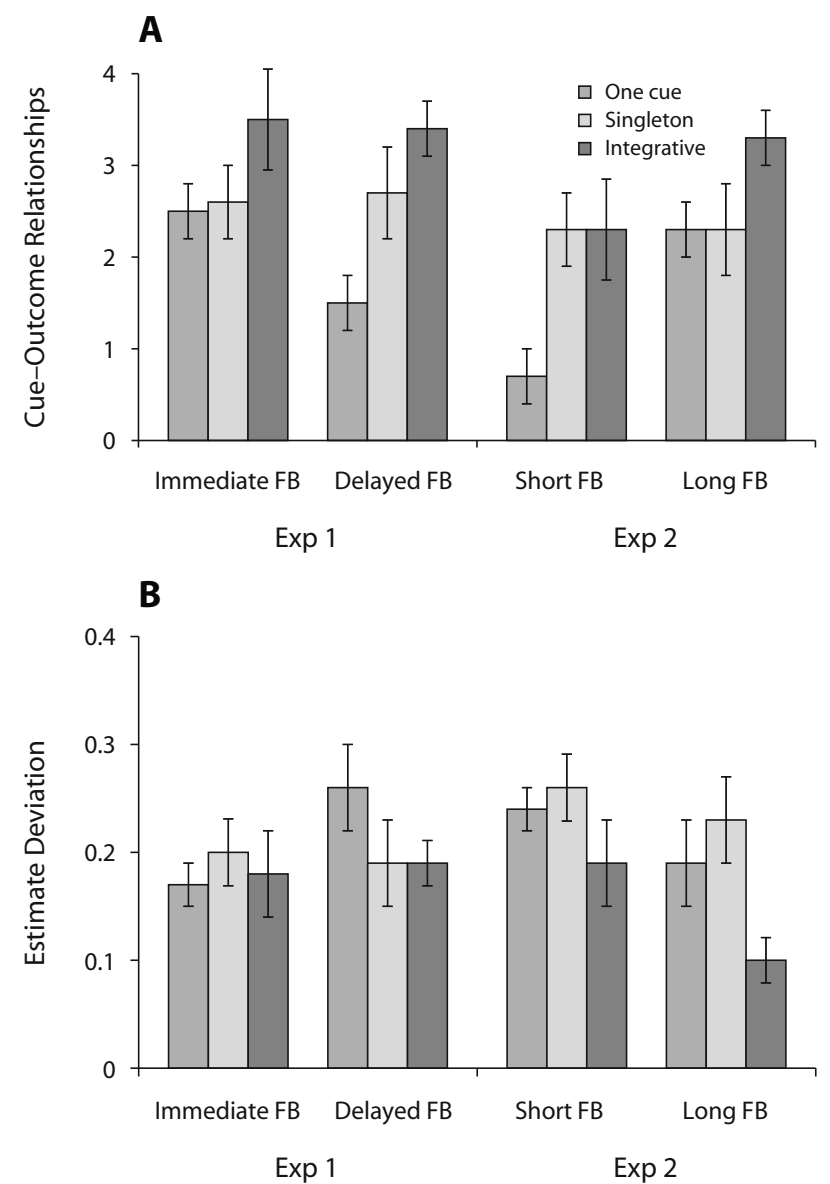

Figure 3. Reported structure awareness by the participants in Experiments 1 and 2 as a function of the response strategy in use during the final block. Participants relying upon an incorrect or random strategy were excluded. (A) Mean numbers of cue-outcome relationships correctly identified by participants. (B) Mean cue weight awareness scores, computed by taking the absolute difference between the actual probability that rain followed a cue and a participant's estimate for that cue, then averaging together the difference scores for all four cues. Lower deviation scores indicate higher levels of structure awareness. FB, feedback. 
response strategy $[F(2,32)=14.22, p<.001]$. Post hoc Tukey HSD tests indicated that the participants who relied on an integrative response strategy were able to correctly identify more cue-outcome relationships than were those participants who relied on a singleton or one-cue strategy; no difference emerged between the participants who used one-cue or singleton strategies. Neither the main effect of condition $(p>.1)$ nor the interaction of condition and strategy type $(p>.2)$ was significant. Together, these data indicated that the participants who relied on an integrative strategy had more extensive declarative knowledge of the WPT structure than did those who relied on a simple strategy. In addition, higher accuracy on the WPT was associated with greater awareness of the individual cueoutcome relationships $[r(37)=.76, p<.001]$ and more accurate cue weight awareness $[r(37)=-.55, p<.001]$, indicating that structure awareness also was associated with classification performance.

\section{Discussion}

The overall results of Experiment 1 indicated that a delay in feedback delivery had no impact on classification accuracy at any point in the task or on the distribution of response strategies in use. Regardless of when feedback occurred, the participants exhibited a similar progression from relying on simple one-cue and singleton strategies to relying on more integrative strategies. These data indicate that the explicit learning processes are sufficient to support a reliance on integrative strategies. This conclusion is further supported by the finding that explicit structure awareness was more extensive for the participants who relied on an integrative strategy, relative to those who relied on simpler strategies.

These findings converge with previous studies of WPT performance that have demonstrated normal WPT performance despite significant delays in feedback delivery (up to $6 \mathrm{sec}$; Aron et al., 2004; Heffernan \& Newell, 2008; Poldrack et al., 2001). Neurobiological theories of category learning (e.g., Ashby \& Valentin, 2005; Shohamy et al., 2008) argue that implicit learning depends on reinforcement-mediated dopamine signals that alter the synaptic connections associated with the immediately preceding stimulus and associated response. If reinforcement is delayed, the dopamine release will modify inappropriate synapses, thereby disrupting implicit learning. Explicit learning is unaffected by a delay in feedback, presumably because the relevant rule can be verbalized and maintained in working memory until feedback occurs (Maddox \& Ashby, 2004).

Consistent with this, feedback delay disrupted information integration category learning, which is thought to depend on implicit processes, but had no impact on explicit, rule-based category learning (Maddox et al., 2003; Maddox \& Ing, 2005). Failure to find any effect of feedback delay here or in other studies suggests that the WPT does not necessitate implicit processes. It is worth noting, however, that the feedback delay manipulation may not have actually disrupted implicit learning processes on the WPT. Whereas the Gabor-patch stimuli used by Maddox and colleagues are difficult to verbally rehearse, the participants could easily have rehearsed the WPT stimuli (e.g., "stars, circles, and squares") during the delay period. In this way, synapses associated with the auditory representation of the stimulus would remain active throughout the delay period. Unfortunately, it is difficult to differentiate between such aural implicit learning and explicit rule learning, since any manipulation designed to disrupt one of these processes would likely disrupt the other. Consequently, it is difficult to determine whether the null effects in the present experiment reflected the absence of implicit learning or a shift in the way the participants implicitly learned the task.

\section{EXPERIMENT 2}

To further examine the relative contributions of implicitand explicit-learning processes in WPT performance, Experiment 2 minimized explicit processes by limiting the time available for the participants to process feedback. Typically, participants are given $2-5 \mathrm{sec}$ to respond to the categorization stimulus and a similar amount of time to process feedback prior to beginning the next trial (e.g., Foerde et al., 2006; Foerde et al., 2007; Gluck et al., 2002; Knowlton et al., 1994). These delays allow participants the time to perform the hypothesis-testing processes necessary for explicit category learning. Here, hypothesis testing was disrupted by requiring the participants to respond within $1 \mathrm{sec}$ after the categorization stimulus appeared and then complete an attention-demanding memory-scanning task immediately following the delivery of feedback. These manipulations have been shown to significantly disrupt explicit category learning, yet have minimal impact on implicit-learning processes (Maddox et al., 2004). As with Experiment 1, performance was assessed through mean classification accuracy and individual response strategies for each block of the task. Measures of structure awareness were also collected once the task had been completed.

\section{Method}

\section{Participants}

Thirty-two undergraduate students participated for partial fulfillment of a class requirement. The participants were randomly assigned to complete either the long-feedback-processing condition (long feedback; $n=16$ ) or the short-feedback-processing condition (short feedback; $n=16$ ).

\section{Materials and Procedure}

Memory scanning. The memory-scanning task followed the procedure outlined by Maddox et al. (2004). On each trial, four digits (0-9) were randomly selected and displayed for $500 \mathrm{msec}$ in 48point type. Each digit appeared at the corner of an imaginary square, 400 pixels in height and width, centered on the screen. Following memory set presentation, a blank screen appeared for 1,000 msec, followed by a single probe digit. The probe was sampled randomly with a .5 probability of having been shown in the memory set. The selected digit was centered on the screen with the question, "Was this one of the numbers you saw?" The participants then responded yes or no by pressing one of two labeled keys that were different from those used in classification learning. The memory-scanning task was emphasized to the participants as the primary task, and they were told to respond as accurately as possible. 
Classification-learning trials. The structure of the WPT was identical to that outlined in Experiment 1, with the following exceptions. Following each classification trial, the participants completed a trial of memory scanning. The procedure for a typical trial within each condition was as follows: Short-feedback condition, classification stimuli appeared/response/500-msec corrective feedback/500-msec memory scan stimulus/1,000-msec delay/ memory scan probe/2,500-msec delay/2,000-msec delay; long feedback condition, classification stimuli appeared/response/500-msec corrective feedback/2,500-msec delay/500-msec memory scan stimulus/1,000-msec delay/memory scan probe/2,000-msec delay. In both conditions, the participants were required to respond to the classification stimulus within $1 \mathrm{sec}$ of its appearance. To ensure that the participants were comfortable responding this quickly, they completed 20 trials each of practice on the classification task by itself, the memory-scanning task by itself, and finally the classification task and memory-scanning task together. On these practice trials, the only feedback provided to the participants on the classification task was an indication of the response they had made ("You responded rain"); they were not informed of whether or not they were correct. Upon finishing the WPT, the participants completed an assessment of structure awareness identical to that outlined in Experiment 1.

\section{Results}

\section{Classification Accuracy}

For each participant, the proportion of optimal responses was computed for each block. Figure 4 illustrates the proportion of optimal responses for each condition as a function of block. Chi-square analysis indicated that a higher proportion of participants in the short-feedback condition $(31 \%)$ than in the long-feedback condition $(0 \%)$ failed to exceed chance levels of performance $\left[\chi^{2}(N=32)=5.93\right.$, $p<.05]$. To further assess the impact of limited feedbackprocessing time, a mixed ANOVA was conducted with learning condition (long feedback vs. short feedback) and block (1-4) as factors. A significant effect of condition indicated that the participants in the short-feedback condition made fewer optimal responses $(M=.55, S E=.10)$ than did those in the long-feedback condition $(M=.63$, $S E=.10)[F(1,30)=5.71, p<.05]$. Across the two con-

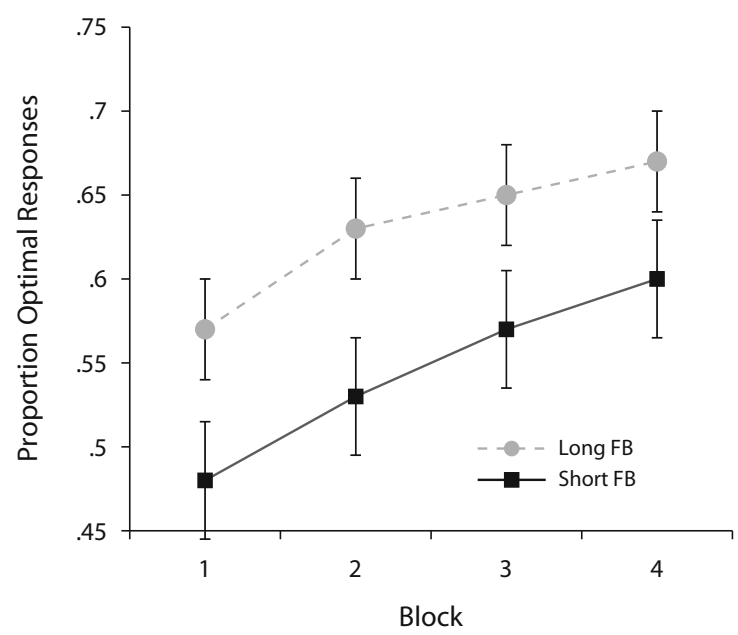

Figure 4. Proportion of optimal responses for each condition of Experiment 2 as a function of block. Error bars represent standard errors of the means. FB, feedback. ditions, classification accuracy improved over the course of the task, as evidenced by a significant main effect of block $[F(3,90)=7.00, p<.001]$. The interaction of block and condition was not significant $(p>.9)$, suggesting that the disruptive effect of limiting feedback-processing time did not dissipate as the task progressed.

Learning on each block was separately assessed for the short- and long-feedback conditions, using one-tailed $t$ tests to determine whether classification accuracy exceeded chance levels ( $50 \%$ accuracy). In the long-feedback condition, mean accuracy exceeded chance on Block 1 $[t(15)=2.50, p<.05]$, Block $2[t(15)=3.70, p<.01]$, Block $3[t(15)=5.95, p<.001]$, and Block $4[t(15)=$ $5.50, p<.001]$. By contrast, performance in the shortfeedback condition exceeded chance only on the final block $[t(15)=2.53, p<.05]$; performance was no better than chance on Block $1[t(15)=0.50, p>.6]$, Block 2 $[t(15)=1.12, p>.2]$, or Block $3[t(15)=2.02, p>.06]$. These analyses indicated that although the participants in the short-feedback condition performed poorly, evidence of learning emerged after considerable training.

\section{Strategy Analysis}

Determination of individual response strategies followed the procedure described in Experiment 1. The distribution of response strategies within each block is shown separately for each condition in Figure 5.

Frequency of inappropriate strategies. An inspection of Figure 4 indicates that a number of participants, especially those in the short-feedback condition, relied on a random or incorrect strategy at some point in the task. As in Experiment 1, the three classes of correct strategies (one cue, singleton, and integrative) were pooled together as a single group of correct strategies. To determine whether the distribution of random, incorrect, and correct strategies varied across the two conditions, a series of chi-square analyses were conducted within each block. On Block 1, the distribution of strategies varied across conditions $\left(\chi^{2}=7.11, p<.05\right)$, reflecting the fact that the participants in the short-feedback condition were more likely than those in the long-feedback condition to rely on an incorrect strategy. The distribution of strategies also varied on Block $2\left(\chi^{2}=5.93, p=.05\right)$, reflecting the fact that the participants in the short-feedback condition were more likely than those in the long-feedback condition to rely on a random strategy; reliance on incorrect strategies was similarly low across the two conditions. Although short-feedback participants were less likely than long-feedback participants to rely on a correct response strategy for Blocks 3 and 4, these trends were not significant ( $p>.2$ in each case).

Given the relatively high incidence of inappropriate strategies, it is worthwhile to examine whether reliance on inappropriate strategies varied over the course of the task. A series of Wilcoxon signed ranks tests, pooled across conditions, indicated that the participants were more likely to rely on correct strategies as the task progressed. Relative to Block 1, reliance on correct strategies was higher in Block $3(z=-2.80, p<.01)$ and Block $4(z=$ $-2.32, p<.05$ ). Similarly, relative to Block 2 , reliance on 

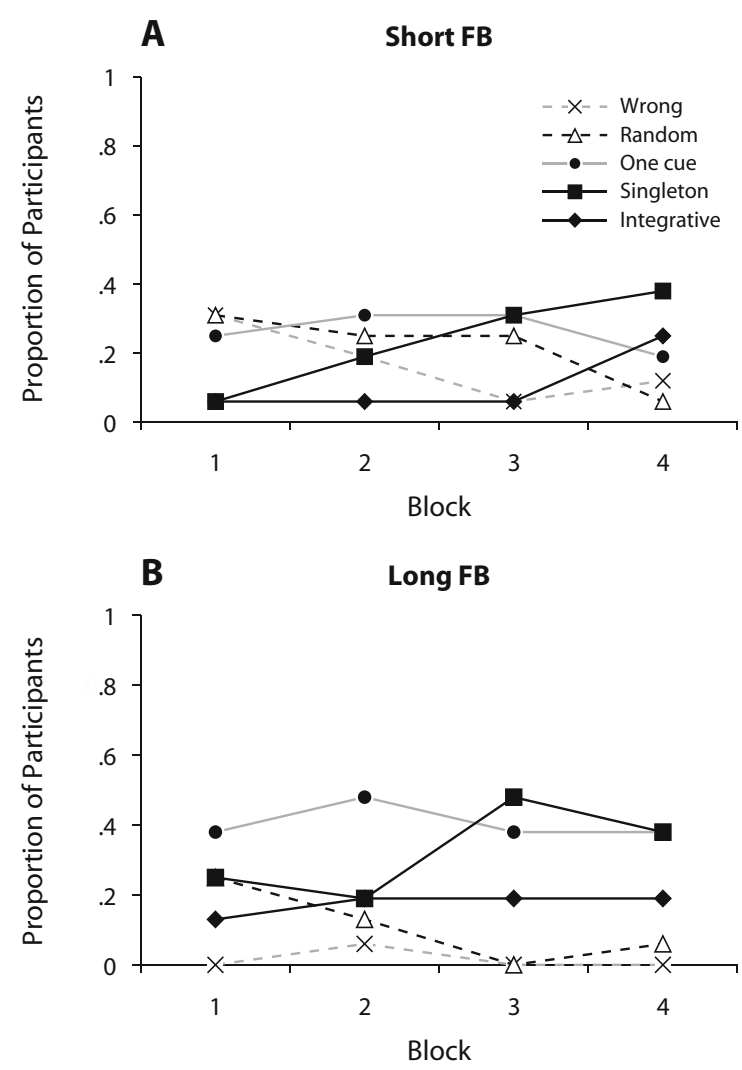

Figure 5. Strategy distribution for the participants in Experiment 2 as a function of block under conditions of short feedback (FB) processing time (A) and long FB processing time (B).

correct strategies was higher in Block $3(z=-1.98, p<$ $.05)$ and Block $4(z=-2.01, p<.05)$. No other paired block comparisons were significant $(p>.1)$. Together, these analyses indicated that the frequency with which the participants relied on inappropriate response strategies was higher in the short-feedback condition but that both groups exhibited increased reliance on correct strategies as the task progressed.

Distribution of correct response strategies. Although a number of participants relied on inappropriate (random or wrong) strategies, it is worthwhile to also examine the distributions of the three types of correct response strategies - namely, singleton, one-cue, and integrative strategies. A series of chi-square analyses conducted within each block indicated that the distribution of correct strategies did not differ across the two conditions for Block $1\left[\chi^{2}(2, N=18)=0.60, p>.7\right]$, Block $2\left[\chi^{2}(2\right.$, $N=22)=0.63, p>.7]$, Block $3\left[\chi^{2}(2, N=27)=0.52\right.$, $p>$.7], or Block $4\left[\chi^{2}(2, N=28)=1.0, p>.7\right]$. Thus, among the participants who relied on correct strategies, feedback processing time had little impact on the particular strategy in use.

To examine whether the distribution of correct strategies varied over the course of the task, response strategies were reclassified as either simple (one-cue and singleton strat- egies) or integrative. A series of Wilcoxon signed ranks tests indicated that reliance on integrative strategies was higher in Block 4 than in Block $1(z=-2.11, p<.05)$; no other block differences in strategy use emerged. The same pairwise block comparisons were conducted on the distribution of singleton versus one-cue strategies, but no differences emerged across any of the blocks $(p>.2$ for each block comparison). These data indicated that across conditions, the participants relied primarily on simple strategies until the final block of the task, at which point a few participants began to rely on integrative strategies.

Relationship of strategy and accuracy. To determine whether the participants in the two feedback conditions differed in the way in which they made use of correct strategies, separate ANOVAs were conducted on mean accuracy within each block, with condition and strategy type (one cue, singleton, and integrative) as factors. No effect of strategy type emerged for Block $1[F(2,18)=3.07$, $p=.08]$ or Block $2[F(2,16)=1.84]$. Performance did vary by strategy type for Block $3[F(2,21)=21.62, p<$ $.001]$ and Block $4[F(2,22)=21.18, p<.001]$; in each of these blocks, post hoc Games-Howell tests indicated that performance was higher for those using an integrative strategy than for those relying on either of the simple strategies; no differences in accuracy emerged between the participants relying on one-cue and singleton strategies. Regardless of block, performance did not vary by feedback-processing condition, nor did the effect of strategy type vary by condition. To summarize, among the participants who relied on correct response strategies, WPT performance was generally unaffected by the amount of time available for feedback processing but varied considerably with the type of strategy in use.

\section{Structure Awareness}

Following the methods outlined in Experiment 1, two measures of structure awareness were created for each participant: (1) number of correctly identified cue-outcome relationships and (2) cue weight awareness, based on the degree to which the participants' cue weight estimates deviated from the true cue weights. In Tables 2 and 3, the participants' awareness of the cue-outcome relationships and underlying cue weights, respectively, are presented for each individual cue as a function of feedback condition.

The participants in the long-feedback condition correctly identified more cue-outcome relationships $(M=$ 2.56, $\mathrm{S} E=0.20)$ than did those in the short-feedback condition $(M=1.56, S E=0.27)[t(30)=2.93, p<.01]$. Similarly, the cue weight estimates provided by the participants in the long-feedback condition $(M=1.93, S E=$ 0.02 ) tended to deviate less from the true cue weights than did the estimates provided by the participants in the shortfeedback condition $(M=0.25, S E=0.02)[t(30)=1.93$, $p<.06]$. Neither of the groups was entirely accurate at estimating the individual cue weights, however. A series of one-sample $t$ tests comparing the actual cue weight with the mean of estimates provided by the participants in the short-feedback condition revealed significant differences for Cue 1 [strong sun; $t(15)=3.97, p<.01$ ], Cue 2 [weak sun; $t(15)=1.97, p<.08]$, Cue 3 [weak rain; $t(15)=$ 
2.34, $p<.05$ ], and Cue 4 [strong rain; $t(15)=6.58, p<$ $.001]$. A similar set of analyses conducted on the cue weight estimates provided by the participants in the longfeedback condition revealed significant differences for Cue $1[t(15)=3.13, p<.01]$, Cue $2[t(15)=2.42, p<$ $.05]$, and Cue $4[t(15)=2.56, p>.5]$; estimates for Cue 3 did not differ from the cue's actual weight $[t(15)=0.58$, $p>.8]$. As is detailed in Table 3, although the cue weight estimates were not especially accurate, those provided by the participants in the long-feedback condition were in the correct direction for all four cues, whereas the mean estimates provided by the participants in the short-feedback condition were in the correct direction for Cue 1 (strong sun card) only.

To better understand the relationship between structure awareness and strategy use, separate ANOVAs were conducted on each measure, with condition (short vs. long feedback) and final block response strategy (one cue, singleton, and integrative) as factors. These analyses indicated that response strategy predicted the level of cue-outcome awareness $[F(2,22)=5.49, p<.05]$ and cue weight awareness $[F(2,22)=3.60, p<.05]$. The participants who relied on an integrative strategy in the final block tended to hold greater awareness of individual cue weights and cue-outcome relationships than did the participants who relied on a one-cue or singleton strategy (see Figure 3); Tukey's HSD tests indicated that these pairwise differences were not significant, however. The effect of strategy type on awareness did not vary with condition, however. The condition $\times$ strategy type interaction was not significant for the cue-outcome measure $(p>.1)$ or the cue weight measure $(p>.7)$.

Awareness was associated not only with the type of response strategy that a participant used in the final block, but also with overall performance on the WPT. Pooled across conditions, higher classification accuracy on the WPT was associated with greater awareness of the individual cue-outcome relationships $[r(31)=.63, p<.01]$ and more accurate knowledge of underlying cue weights $[r(31)=-.56, p<.01]$. These relationships were driven primarily by the participants in the long-feedback condition [cue-outcome awareness, $r(15)=.61, p<.05$; cue-weight estimate, $r(15)=-.57, p<.05]$; no relationships emerged within the short-feedback condition [cueoutcome awareness, $r(15)=.31, p>.3$; cue weight estimate, $r(15)=-.27, p>.3$ ].

In summary, these data indicated that structure awareness was greater among those participants who received time to explicitly process feedback. Furthermore, structure awareness, which was associated with WPT accuracy, was higher for those participants who relied on an integrative response strategy during the final classification block, relative to those who relied on a simpler strategy.

\section{Discussion}

The results of Experiment 2 indicated that WPT learning was slower and less robust when explicit processing of feedback was minimized. The negative impact of reduced feedback processing was apparent early in the WPT and remained as the task progressed, with roughly one third of the participants in the short-feedback condition failing to exceed chance levels of performance by the final block. Disruption of feedback processing also increased the participants' tendency to rely on inappropriate response strategies and reduced their level of structure awareness. Furthermore, WPT learning was correlated with structure awareness, but only when feedback-processing time was sufficient to support explicit-learning processes. These findings emphasize the necessity of explicit processes in WPT performance.

It is worth noting that although performance in the short-feedback condition was especially poor, performance in the long-feedback condition was not especially strong either. Whereas the participants in Experiment 1 averaged $68 \%$ optimal responses, those in the longfeedback condition in this experiment averaged only $63 \%$ optimal responses, suggesting that learning was hampered in both conditions in Experiment 2. Similarly, Maddox et al. (2004) examined the impact of disrupting feedback processing on rule-based and information integration tasks and found that a sizable percentage of participants failed to exceed chance levels of performance in the longfeedback (31\%) and short-feedback (41\%) conditions of a rule-based task. By contrast, very few participants $(8 \%)$ failed to exceed chance on an information integration task, regardless of whether feedback processing was disrupted. It is likely that the requirement to respond rapidly $(<1 \mathrm{sec}$ of stimulus appearance) limited the participants' ability to perform hypothesis-testing processes prior to responding. Thus, the relatively poor performance of the participants in this experiment further supports the argument that the WPT relies on explicit hypothesis-testing processes.

\section{GENERAL DISCUSSION}

In this study, the contributions of implicit and explicit category-learning processes to WPT performance were examined. In Experiment 1, disruption of implicit learning processes by a delay in feedback had no effect on classification performance or the distribution of individual response strategies. In Experiment 2, disrupting explicitlearning processes by limiting feedback-processing time had a substantial impact on WPT performance. When explicit hypothesis testing was disrupted, the participants exhibited only a minimal amount of learning and were less likely than others to rely on a correct response strategy. In addition, strategy analyses in both experiments indicated that both implicit and explicit processes were sufficient to support simple, one-cue, or singleton strategies, as well as more complex integrative strategies. These findings challenge the idea (e.g., Shohamy et al., 2008) that the use of particular types of strategies reflects a specific reliance on either implicit- or explicit-learning processes.

The present pattern of findings suggests that WPT learning was driven primarily by declarative processes. However, although the participants in the short-feedback condition performed poorly, they did exhibit learning after considerable training. It is possible that disrupting feedback processing hampered, but did not eliminate, explicit-learning processes. Previous work with explicit, 
rule-based category structures showed that when feedback processing was disrupted, participants required greater training to acquire the optimal, rule-based strategy (Maddox et al., 2004). Similarly, many participants in the short-feedback processing condition ultimately arrived at a successful strategy after extended training. Thus, late improvement in WPT performance may reflect the slowed development of explicit knowledge.

Alternatively, it is possible that performance improvements in the short-feedback condition reflected the late emergence of implicit knowledge. The participants may have developed implicit knowledge throughout the task but were unable to express it due to initial persistence with inappropriate response strategies. Elsewhere, reliance on inappropriate explicit strategies hampered the expression of implicit knowledge (Howard \& Howard, 2001; Jitsumori, 1993; Maddox \& Ashby, 2004; Maddox et al., 2003). Performance improves once participants abandon their unsuccessful response strategies and begin to rely on implicit knowledge. Alternatively, the late improvements in WPT performance could indicate that the development of implicit knowledge requires extensive practice. However, learning of information integration category structures, which is thought to rely on implicit processes, developed within the first block of 80 trials, even when feedback processing was disrupted (Maddox et al., 2004; Waldron \& Ashby, 2001; Zeithamova \& Maddox, 2006).

If implicit category learning requires extensive training, disrupting implicit processes through a delay in feedback, as in Experiment 1, may impact performance only after considerable training. Consequently, the WPT used here could have been too brief to support the intended assessment of implicit processes. Although this possibility cannot be ruled out, it runs counter to previous claims of implicit knowledge expression in shorter versions of the task, which included roughly 100 classification trials (Aron et al., 2005; Foerde et al., 2006; Poldrack et al., 2001; Poldrack et al., 1999). Moreover, feedback delay disrupts information integration category learning within the first block of 80 trials (Maddox et al., 2003; Maddox \& Ing, 2005). In contrast, these same studies showed that delayed feedback had no impact on explicit, rule-based category-learning performance, even when training was extended to 300 trials. Consequently, it is unlikely that extending the length of the WPT would have eventually revealed evidence of implicit category knowledge.

Instead, the pattern of classification performance across the two experiments emphasizes the importance of explicit processes for WPT performance. This possibility is further supported by evidence that classification performance and response strategy were strongly associated with explicit awareness of the underlying strategy. Specifically, the participants who relied on an integrative strategy, which most closely approximates the optimal response strategy, tended to be those who verbalized greater structure awareness. ${ }^{5}$ These results converge nicely with previous behavioral research pointing to the necessity of attentional resources for WPT learning. Performance of an attention-demanding secondary task has been shown to disrupt WPT performance, but the nature of this disruption has varied across studies (for impaired WPT learning, see Heffernan \& Newell, 2008; Newell et al., 2007; for normal WPT learning, see Foerde et al., 2006; Foerde et al., 2007). Specifically, the participants learned normally when the secondary task primarily taxed working memory resources during stimulus presentation but had difficulty when a secondary task demanded working memory throughout the entire trial, including the period in which feedback was processed. In conjunction with the present findings, these results suggest that normal WPT learning develops only when sufficient attentional resources are available to support effective feedback processing. Collectively, these results suggest that WPT learning depends primarily on explicit, rule-based processes.

Importantly, this behavioral evidence suggests a reinterpretation of neuroimaging evidence regarding WPT performance. In particular, as participants progress through the WPT, striatal activation increases (Aron et al., 2005; Poldrack et al., 2001; Seger \& Cincotta, 2005); this has typically been interpreted as indicating the operation of nondeclarative learning processes, but the present results challenge this assumption. Considerable evidence points to the necessity of striatal activity in both explicit (e.g., Ell, Marchant, \& Ivry, 2006; Eslinger \& Grattan, 1993; Nomura et al., 2007) and implicit (e.g., Nomura et al., 2007) category learning. Thus, one cannot interpret striatal activation during a category-learning task as evidence that the task specifically demands implicit, or explicit, processes (Ashby \& Valentin, 2005). Given accumulating evidence pointing to a necessary role for declarative-learning processes in WPT performance, it is reasonable to conclude that striatal activity during WPT performance reflects a reliance on explicit, rule-based learning. Future neuroimaging examinations of the task should include manipulations, such as those used here, or particular category structures (e.g., rule-based vs. information integration structures) that have been previously demonstrated to specifically emphasize either explicit- or implicit-learning processes. This would help to distinguish the nature of the neural activity that supports each of these processes.

Although the WPT is often referred to as a measure of nondeclarative learning, it does not fit with theoretical conceptualizations of nondeclarative category learning (e.g., Ashby et al., 1998; Maddox \& Ashby, 2004). Specifically, implicit category-learning tasks are optimally performed using a strategy that is not readily verbalized, are impaired by a delay in feedback, and are unaffected by disruptions to hypothesis-testing processes, due to experimental manipulations or declines in executive function. Evidence here and elsewhere has indicated that the WPT fails to satisfy these conditions (Gluck et al., 2002; Knowlton et al., 1996; Lagnado et al., 2006; Meeter et al., 2006; Newell et al., 2007; Price, 2005, 2006; Reber et al., 1996). Several recent articles have argued that the pattern of evidence regarding the WPT is consistent with the existence of a single, explicit category-learning system (Lagnado et al., 2006; Newell et al., 2007; Zaki, 2004). However, the conceptualization of category learning as depending on a unitary, explicit system is difficult to 
reconcile with the accumulating number of functional, neurobiological, and neuropsychological dissociations between rule-based tasks, which are optimally solved using explicit hypothesis-testing processes, and information integration tasks, which are optimally solved using complex, nonverbalizable strategies (for a relevant review, see Maddox \& Ashby, 2004). Presently, it is unclear how a unitary system of category learning could explain these dissociations, each of which are consistent with multiplesystem accounts of category learning.

One of the most successful multiple-system models of category learning is the competition between verbal and implicit systems (COVIS) model (Ashby et al., 1998; Ashby \& Valentin, 2005; Maddox \& Ashby, 2004). Central to the COVIS model is the idea that the implicit and explicit systems operate competitively and that responding is governed by the system producing the most strongly weighted response. It is further argued that the explicit system predominates, especially during the initial phase of a task, even if the resulting responses are inappropriate. If the verbal system fails to support reasonable levels of success, responding should increasingly reflect the contributions of implicit systems. Consistent with this, the learning of information integration categories improves when participants are placed in situations designed to disrupt hypothesis-testing processes (Maddox, Baldwin, \& Markman, 2006). This did not occur here; the participants in the short-feedback condition were more likely than others to have persisted with inappropriate strategies. This pattern has been observed elsewhere. Under certain conditions, participants persist with suboptimal explicit strategies despite the fact that a shift toward more implicit processes would be advantageous (Maddox et al., 2006). It may be that on the WPT, the probabilistic nature of feedback results in periodic reinforcement of inappropriate strategies, thereby reducing the likelihood that participants will abandon such strategies.

Although the WPT is commonly viewed as a test of nondeclarative learning, the present results call such an assumption into question. The WPT has been used extensively to examine the neural mechanisms of nondeclarative learning (Aron et al., 2005; Foerde et al., 2006; Poldrack et al., 2001; Poldrack et al., 1999; Shohamy et al., 2004) and to assess the integrity of nondeclarative learning processes in various neuropsychological populations (Beninger et al., 2003; Eldridge et al., 2002; Foerde et al., 2008; Hopkins et al., 2004; Kéri et al., 2002; Knowlton et al., 1996; Knowlton et al., 1994). However, the present study suggests that nondeclarative learning processes are not necessary and are insufficient for the levels of performance commonly seen with the WPT. Instead, classification appears to draw heavily on attention-demanding explicit-learning strategies, including hypothesis testing. In conjunction with other findings, the present results suggest a reinterpretation of the neuropsychological and neuroimaging data gathered using the WPT. More generally, these results emphasize the importance of behavioral research to support careful examination of tasks used in the cognitive neuroscience field.

\section{AUTHOR NOTE}

Correspondence concerning this article should be addressed to A. L. Price, Department of Psychology, Elizabethtown College, Elizabethtown, PA 17022 (e-mail: pricea@etown.edu).

\section{REFERENCES}

Arbuthnott, G. W., Ingham, C. A., \& Wickens, J. R. (2000). Dopamine and synaptic plasticity in the neostriatum. Journal of Anatomy, 196, 587-596.

Aron, A. R., Gluck, M. A., \& Poldrack, R. A. (2005). Long-term test-retest reliability of functional MRI in a classification learning task. NeuroImage, 29, 1000-1006.

Aron, A. R., Shohamy, D., Clark, J., Myers, C., Gluck, M. A., \& PoldRACK, R. A. (2004). Human midbrain sensitivity to cognitive feedback and uncertainty during classification learning. Journal of Neurophysiology, 92, 1144-1152.

Ashby, F. G., Alfonso-Reese, L. A., Turken, A. U., \& Waldron, E. M. (1998). A neuropsychological theory of multiple systems in category learning. Psychological Review, 105, 442-481.

Ashby, F. G., \& VAlentin, V. V. (2005). Multiple systems of perceptual category learning: Theory and cognitive tests. In H. Cohen \& C. Lefebvre (Eds.), Categorization in cognitive science (pp. 548-573). New York: Elsevier.

Beninger, R., Wasserman, J., Zanibi, K., Carbonneau, D., ManGels, J., \& Beninger, B. (2003). Typical and atypical medications differentially affect two nondeclarative memory tasks in schizophrenic patients: A double dissociation. Schizophrenic Research, 61, 281-292.

Cohen, N., \& Eichenbaum, H. (2001). From conditioning to conscious recollection: Memory systems of the brain. New York: Oxford University Press.

DeCaro, M. S., Thomas, R. D., \& Beilock, S. L. (2008). Individual differences in category learning: Sometimes less working memory capacity is better than more. Cognition, 107, 284-294.

Eldridge, L. L., Masterman, D., \& Knowlton, B. J. (2002). Intact implicit habit learning in Alzheimer's disease. Behavioral Neuroscience, 116, 722-726.

Ell, S. W., Marchant, N. L., \& Ivry, R. B. (2006). Focal putamen lesions impair learning in rule-based, but not information-integration categorization tasks. Neuropsychologia, 44, 1737-1751.

Eslinger, P. J., \& Grattan, L. M. (1993). Frontal lobe and frontalstriatal substrates for different forms of human cognition. Neuropsychologia, 31, 17-28.

Foerde, K., Knowlton, B. J., \& Poldrack, R. A. (2006). Modulation of competing memory systems by distraction. Proceedings of the $\mathrm{Na}$ tional Academy of Sciences, 103, 11778-11783.

Foerde, K., Poldrack, R. A., \& Knowlton, B. J. (2007). Secondarytask effects on classification learning. Memory \& Cognition, 35, 864874.

Foerde, K., Poldrack, R. A., Knowlton, B. J., Sabb, F. W., Bookheimer, S. Y., Bilder, R. M., Et AL. (2008). Selective corticostriatal dysfunction in schizophrenia: Examination of motor and cognitive skill learning. Neuropsychology, 22,100-109.

Gluck, M. A., Shohamy, D., \& Myers, C. E. (2002). How do people solve the "weather prediction" task? Individual variability in strategies for probabilistic category learning. Learning \& Memory, 9, 408-418.

HeFFERNAN, M., \& NeWELl, B. (2008). Secondary tasks disrupt performance in probabilistic categorization, but delayed feedback does not: Implications for multiple system accounts. Unpublished manuscript, University of New South Wales.

Hollerman, J. R., \& Schultz, W. (1997). Dopamine neurons report an error in the temporal prediction of reward during learning. Nature Neuroscience, 1, 304-308.

Hopkins, R. O., Myers, C. E., Shohamy, D., Grossman, S., \& Gluck, M. A. (2004). Impaired probabilistic category learning in hypoxic subjects with hippocampal damage. Neuropsychologia, 42, 524-535.

Howard, D. V., \& Howard, J. H., JR. (2001). When it does hurt to try: Adult age differences in the effects of instructions on implicit pattern learning. Psychonomic Bulletin \& Review, 8, 798-805.

JiménEZ, L., \& VÁZQuez, G. A. (2005). Sequence learning under dual- 
task conditions: Alternatives to a resource-based account. Psychological Research, 69, 352-368.

JITSUMORI, M. (1993). Category discrimination of artificial polymorphous stimuli based on feature learning. Journal of Experimental Psychology: Animal Behavior Processes, 19, 244-254.

Kéri, S., Szlobodnyik, C., Benedek, G., Janka, Z., \& GÁdoros, J. (2002). Probabilistic classification learning in Tourette syndrome. Neuropsychologia, 40, 1356-1362.

KerR, J. N. D., \& Wickens, J. R. (2001). Dopamine D-1/D-5 receptor activation is required for long-term potentiation in the rat neostriatum in vitro. Journal of Neurophysiology, 85, 117-124.

Knowlton, B. J., Mangels, J. A., \& Seuire, L. R. (1996). A neostriatal habit learning system in humans. Science, 273, 1399-1402.

Knowlton, B. J., Squire, L. R., \& Gluck, M. A. (1994). Probabilistic category learning in amnesia. Learning \& Memory, 1, 106-120.

Lagnado, D. A., Newell, B. R., Kahan, S., \& Shanks, D. R. (2006). Insight and strategy in multiple cue learning. Journal of Experimental Psychology: General, 135, 162-183.

Maddox, W. T., \& Ashby, F. G. (2004). Dissociating explicit and procedural-learning based systems of perceptual category learning. Behavioural Processes, 66, 309-332.

Maddox, W. T., Ashby, F. G., \& BohIL, C. J. (2003). Delayed feedback effects on rule-based and information-integration category learning. Journal of Experimental Psychology: Learning, Memory, \& Cognition, 29, 650-662.

Maddox, W. T., Ashby, F. G., Ing, A. D., \& Pickering, A. D. (2004). Disrupting feedback processing interferes with rule-based but not information integration category learning. Memory \& Cognition, 32 , 582-591.

Maddox, W. T., Baldwin, G. C., \& Markman, A. B. (2006). A test of the regulatory fit hypothesis in perceptual classification learning. Memory \& Cognition, 34, 1377-1397.

Maddox, W. T., \& Filoteo, J. V. (2001). Striatal contribution to category learning: Quantitative modeling of simple linear and complex nonlinear rule learning in patients with Parkinson's disease. Journal of the International Neuropsychological Society, 7, 710-727.

Maddox, W. T., \& ING, A. D. (2005). Delayed feedback disrupts the procedural-learning system but not the hypothesis testing system in perceptual category learning. Journal of Experimental Psychology: Learning, Memory, \& Cognition, 31, 100-107.

Meeter, M., Myers, C. E., Shohamy, D., Hopkins, R. O., \& GlucK, M. A. (2006). Strategies in probabilistic categorization: Results from a new way of analyzing performance. Learning \& Memory, 13, 230-239.

Newell, B. R., Lagnado, D. A., \& Shanks, D. R. (2007). Challenging the role of implicit processes in probabilistic category learning. Psychonomic Bulletin \& Review, 14, 505-511.

Nomura, E. M., Maddox, W. T., Filoteo, J. V., InG, A. D., Gitelman, D. R., PARrish, T. B., ET AL. (2007). Neural correlates of rule-based and information-integration visual category learning. Cerebral Cortex, 17, 37-43.

Nosofsky, R. M., \& Johansen, M. K. (2000). Exemplar-based accounts of "multiple-system" phenomena in perceptual categorization. Psychonomic Bulletin \& Review, 7, 375-402.

Poldrack, R., Clark, J., Paré-Blagoev, E. J., Shohamy, D., Creso Moyano., J., Myers, C., \& Gluck, M. (2001). Interactive memory systems in the human brain. Nature, 414, 546-550.

Poldrack, R. A., \& PACKARD, M. G. (2003). Competition among multiple memory systems: Converging evidence from animal and human brain studies. Neuropsychologia, 41, 245-251.

Poldrack, R. A., Prabakharan, V., Seger, C., \& Gabrieli, J. D. E. (1999). Striatal activation during cognitive skill learning. Neuropsychology, 13, 564-574.

PrICE, A. (2005). Cortico-striatal contributions to category learning: Dissociating the verbal and implicit systems. Behavioral Neuroscience, 119, 1438-1447.

PrICE, A. (2006). Explicit category learning in Parkinson's disease: Deficits related to impaired rule generation and selection processes. Neuropsychology, 20, 249-257.

Reber, P., Knowlton, B., \& SQuire, L. (1996). Dissociable properties of memory systems: Differences in the flexibility of declarative and nondeclarative memory. Behavioral Neuroscience, 110, 861-871.
Schultz, W. (1992). Activity of dopamine neurons in the behaving primate. Seminars in Neuroscience, 4, 129-138.

Seger, C. A., \& Cincotta, C. M. (2005). The roles of the caudate nucleus in human classification learning. Journal of Neuroscience, 25, 2541-2551.

Shohamy, D., Myers, C. E., Grossman, S., Sage, J., Gluck, M. A., \& PoldRACK, R. A. (2004). Cortico-striatal contributions to feedbackbased learning: Converging data from neuroimaging and neurophysiology. Brain, 127, 851-859.

Shohamy, D., Myers, C. E., Kalanithi, J., \& Gluck, M. A. (2008). Basal ganglia and dopamine contributions to probabilistic category learning. Neuroscience \& Biobehavioral Reviews, 32, 219-236 .

Smith, E. E., Patalano, A., \& Jonides, J. (1998). Alternative strategies of categorization. Cognition, 65, 167-196.

SQuire, L. (1992). Declarative and nondeclarative memory: Multiple brain systems supporting learning and memory. Journal of Cognitive Neuroscience, 4, 232-243.

WALDron, E. M., \& Ashby, F. G. (2001). The effects of concurrent task interference on category learning: Evidence for multiple category learning systems. Psychonomic Bulletin \& Review, 8, 168-176.

ZAKI, S. R. (2004). Is categorization performance really intact in amnesia? A meta-analysis. Psychonomic Bulletin \& Review, 11, 1048-1054.

Zeithamova, D., \& Maddox, W. T. (2006). Dual-task interference in perceptual category learning. Memory \& Cognition, 34, 387-398.

Zeithamova, D., \& MADDOX, W. T. (2007). The role of visuospatial and verbal working memory in perceptual category learning. Memory \& Cognition, 35, 1380-1398.

\section{NOTES}

1. The terms explicit and declarative are used interchangeably here to refer to learning that involves intent to learn and awareness of the learned information. Similarly, implicit and nondeclarative are used interchangeably to refer to learning that occurs incidentally and without awareness of the learned information.

2. These probabilities were obtained by calculating the probability of rain given each particular cue. The conditional probabilities were computed by calculating the probability that rain would occur following a particular cue, divided by the total probability that the cue would occur, regardless of the outcome. For example, as one can calculate from Table 1, in the case of Cue 1, the probability that Cue 1 would be present and that rain could occur $[P($ cue combination $) \times P($ rain $)]$ is calculated by summing across Patterns $8-15(.346)$; the total probability that Cue 1 would occur regardless of the outcome equals the sum of each $P$ (cue combination) for Patterns 8-15 (.462). Thus, the association of Cue 1 with rain can be calculated by computing the probability that rain would occur following Cue $1(.346)$ and dividing by the total probability that Cue 1 would occur (.462). This value is . $346 / .462$, or $75 \%$.

3. Analysis of response strategies initially differentiated between intermediate strategies (Meeter et al., 2006), defined as the singleton plus and $2 v 1$ strategies, and optimal strategies, defined as the all but $2 v 1$ and multicue strategies. However, only $2 \%$ of the participants relied on an optimal strategy in the final block of trials. To increase the power of the strategy analyses, these participants were combined with the $36 \%$ of participants who were relying on an intermediate strategy.

4. The number of participants included in each analysis varied because the participants who relied on a random or wrong response strategy on that block were excluded from that particular analysis.

5. Although these relationships were not statistically significant among the participants in the short-feedback condition, they were in the predicted directions; higher accuracy was associated with greater cue-outcome awareness and more accurate cue weight estimates. In addition, the participants in the short-feedback condition who performed above chance in the final block were relying primarily on simple strategies (singleton, one cue). The participants who relied on these simple strategies were most likely aware of some aspects of the categorization structure but, nevertheless, exhibited poor structure awareness, perhaps because both awareness measures collapsed across all four cues.

(Manuscript received March 24, 2008; revision accepted for publication September 24, 2008.) 